\title{
Characteristics of scour and flow field beneath a forced vibrating circular cylinder
}

\author{
Y.-M. Chiew \& S.-C. Hsieh \\ School of Civil \& Environmental Engineering, Nanyang Technological University, Singapore \\ Y. M. Low \\ Department of Civil \& Environmental Engineering, National University of Singapore, Singapore
}

\begin{abstract}
The objective of the study is to enhance the understanding of scour under a steel catenary riser (SCR), in particular the influence of the SCR dynamic motions on scour. The motion of an SCR could be attributed to the motions of the attached floating platform induced by surface waves and/or currents. In this study, a circular cylinder mounted elastically on a forced vibrating rod was installed and three vibration frequencies were tested to simulate this phenomenon. Flow visualization and high time-resolved PIV measurement techniques were applied to observe and measure the flow field around the cylinder. The results show that there are two distinct types of scour processes, depending on the imposed vibration frequency even though they have the same amplitude. Based on the qualitative observation and quantitative measurement of the flow field and turbulence characteristics, the formation and transmission of the vortices are discussed in this paper.
\end{abstract}

\section{INTRODUCTION}

The formation of a local scour hole around submarine pipelines is an important concern in design because it can lead to fatigue failure due to vortexinduced vibrations; a topic that has been studied extensively over the past 4 - 5 decades. Another type of marine structure similar to a pipeline is the steel catenary riser (SCR). The touch down position of an SCR is also susceptible to scour, but the scour hole developed here differs from that related to a horizontal stationary pipeline in that it moves up and down on the seabed due to the motions of the floating platform. Thus published scour prediction equations for pipelines are inappropriate for SCR design. This is because SCR motions are expected to exacerbate the scouring process due to increased agitation of the sediments on the seabed.

Most previous researches had focussed on the interaction between a 2-dimensional vibrating riser and soil of sea bed under a range of amplitudes and velocities of vertical motion (Aubeny et al., 2008, Clukey et al., 2008, Langford \& Aubeny, 2008, and Hodder et al., 2009). Hodder \& Byrne (2010) carried out experiments to investigate the 3-dimensional interaction between a forced vibrating SCR and a sand bed in still water. They measured the bending moment along the SCR, as well as the sand bed reaction forces and excess water pressure for both monotonic and cyclic tests, while the trench profile was observed qualitatively. Hu et al. (2011) and Westgate et al. (2013) embedded a forced cyclic vibrating riser in a clay seabed and investigated the effect of soil strength, riser displacement rate and loading mode on riser-soil interaction.

Consequently, these previous studies may not reveal the actual scouring mechanism around SCRs. Li et al. (2013) conducted experiments to study the impact of a vibrating SCR on scour in a steady current. A forced vertical vibration of the riser was used in the tests. Their results showed that the scour profiles for a stationary and a vibrating riser were different. The maximum scour depth increased significantly with both vibration amplitude and frequency, with the latter having a more profound influence.

Notwithstanding the limited published studies pertaining to scour around a forced vibrating riser in still water, there is as yet no detailed quantitative study on the interaction between the flow field, scour and vibrating riser. This study aims to obtain a better understanding of the characteristics of the flow field around a vibrating riser and its relationship with the surrounding scour hole. To this end, a physical experiment with a 2-dimensional circular cylinder mounted elastically on a movable rod near an erodible seabed in still water was conducted. A server motor was used to control the movable rod vertically with fixed amplitude and three frequencies in order to simulate the vibration induced by the floating platform.

Flow visualizations and high time-resolved PIV (particle image velocimetry) measurement techniques were applied to observe and measure the flow field around the cylinder. In order to improve the 
measurement accuracy of the velocity both in the space and time domains, a new high time-resolved PIV measurement technique was adopted in the present study. This PIV system used high speed camera instead of the conventional CCD camera to obtain a sufficiently high sampling rate for the velocity and turbulence measurements. A high-pass filter process and multi-time interval method were used to increase the accuracy of the velocity calculation near the scoured sand bed. The characteristics of interactions between the vibrating cylinder, flow field and scour hole during the scouring process are discussed in this paper.

\section{EXPERIMENTAL SETUP}

\subsection{Water flume, sand bed and coordinate system}

Laboratory experiments were carried out in a recirculating flume in the Hydraulics Modeling Laboratory at Nanyang Technological University. The test section, with length $=1100 \mathrm{~cm}$, width $=60 \mathrm{~cm}$ and depth $=60 \mathrm{~cm}$, has glass walls on both sides and the bottom to allow visual observations and PIV measurements.

For the experiments with an erodible bed, one uniform sand with median grain size $d_{50}=0.449 \mathrm{~mm}$ and geometric standard deviation $\sigma_{g}=1.296$ was used. The sand bed was $8.4 \mathrm{~m}$ long with the thickness of $10 \mathrm{~cm}$. The water depth is kept constant at 40 $\mathrm{cm}$. It must be stated that the water remains stationary in all the tests.

Figure 1 shows the coordinate system adopted in this study. The origin is located on the original sand bed level and directly beneath the center of the cylinder. The $x$-axis is in the streamwise direction, while the $y$-axis in the vertical direction.

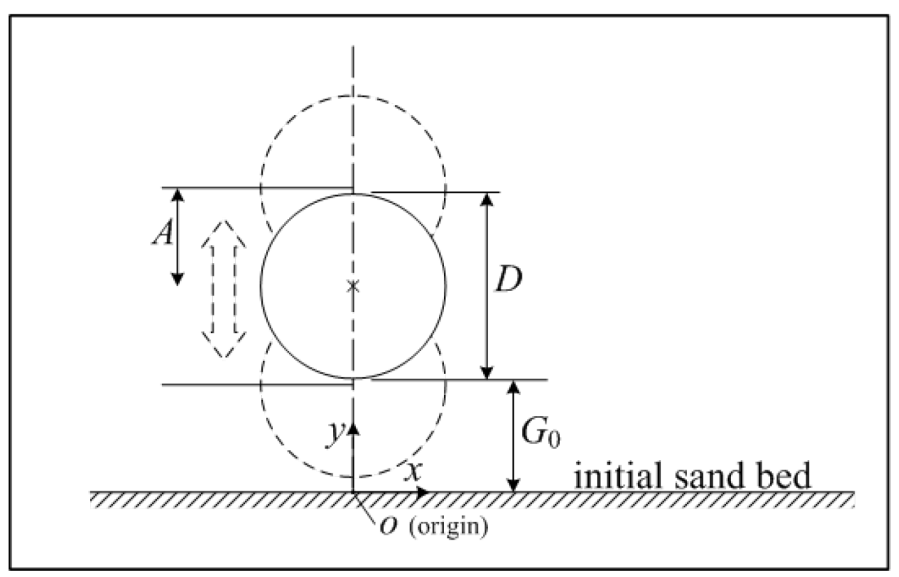

Figure 1. Coordinate system adopted in present study.

\subsection{Test model and vibrating system}

An acrylic circular cylinder with diameter $=3.5 \mathrm{~cm}$, and length $=56 \mathrm{~cm}$ were used as the model in the experiments. The purpose of this study is to explore the characteristics of the flow field around a twodimensional forced vibrating steel catenary riser (SCR) that is elastically mounted and moves vertically, and its relationship with the surrounding scour hole. To this end, the cylinder was mounted on an aluminum supporting frame, which, in turn, is connected by two springs to a moveable rod on another frame that is fixed on the two sides of the flume, as illustrated in Figure 2. A server motor was used to control the movable rod vertically with certain frequency and amplitude in order to simulate the vibration induced by the floating platform. The supporting frame is designed to vibrate only along the vertical direction with the use of four bearings; moreover, the length of rod of the supporting frame can be adjusted for a predetermined gap size between the cylinder and sea bed.

The weight of the vibrating system, including the circular cylinder and the aluminum frame is 1.445 $\mathrm{kg}$, which corresponds to an equivalent mass ratio $m^{*}=2.682$. By conducting free decay tests in still water following the procedure in Sumer and Fredsøe (1997), the following parameters are obtained: natural frequency $f_{N}=0.82 \mathrm{~Hz}$, damping ratio $\zeta=0.124$, and $m * \zeta=0.333$.

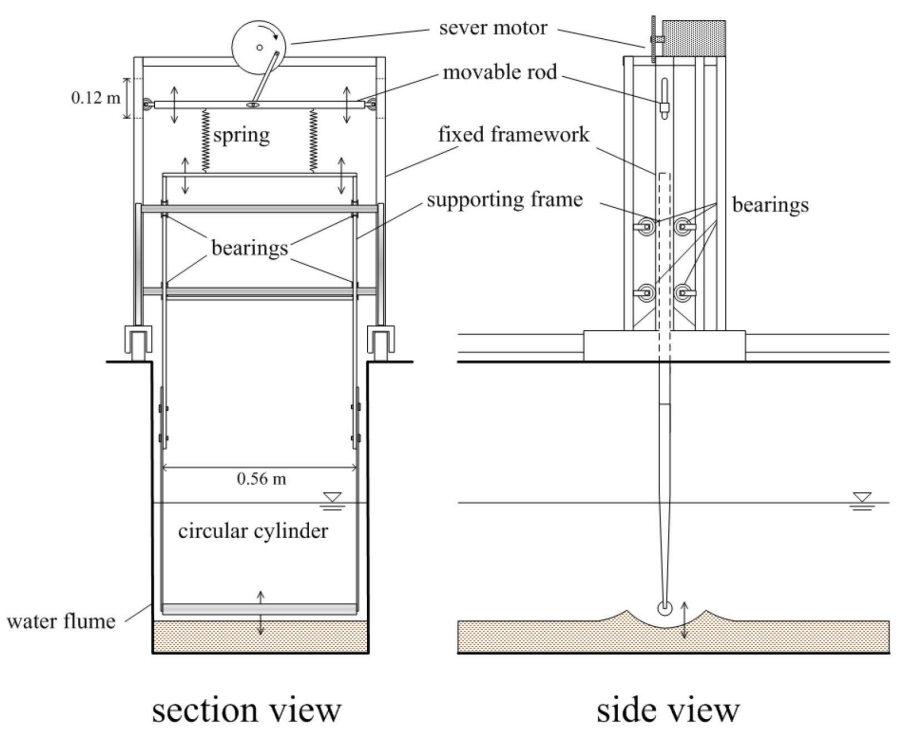

Figure 2. Schematic diagrams of experimental setup.

\subsection{Velocity measurements and analysis techniques}

Although PIV techniques and related applications have become mature in the past decade, there are still some limitations that need to be resolved for the present study. Consequently, a new high timeresolved PIV measurement technique proposed by Hsieh (2008) was adopted in the present study.

The PIV measuring system comprised a $5 \mathrm{~W}$ aircooled laser with wavelength $=532 \mathrm{~nm}$ as the light source, and a high speed camera (Phantom Miro M120) to capture the images. The beam emitted from the laser source was reflected by a mirror, resulting in a laser light fan with $1.5 \mathrm{~mm}$ thickness 
cast downward into the water through a transparent acrylic sheet on the water surface, which reduced reflection from water surface oscillations. Additionally, aluminum particles with diameter $=10 \mu \mathrm{m}$ and specific density $=2.7$ were used as seeding particles in the experiment. Using Stoke's law, the settling velocity of the aluminum particles was estimated to be less than $10 \mu \mathrm{m} / \mathrm{s}$.

The high speed camera with a three-gigabyte memory storage and $1600 \times 1200$ pixel resolution was used to capture images of the particle-laden flow. A $60-\mathrm{mm}$ focal lens was mounted on the high speed camera that was operated at $200 \mathrm{fps}$ (frame per second). A multi-grid processing algorithm was adopted to enhance the accuracy of velocity calculation; the interrogation window started from $32 \times 32$ pixels and ended with $8 \times 8$ pixels.

Moreover, a high-pass filter technique and multitime interval method was also applied to reduce the error from light reflection and time interval selection. Details of this PIV technique can be found in Hsieh (2008). Moreover, wavelet transform was used to phase-average analyses for periodic variations of the flow field.

\section{RESULTS}

The present experimental setup is able to fulfill the objective of studying interactions between seabed scour and a forced vibrating cylinder mounted elastically on an oscillating rod with different frequencies. In this study, only the flow characteristics in the intermediate region $\left(G_{0} / D=1\right)$ around a twodimensional freely vibrating circular cylinder near a plane boundary were investigated. Only one amplitude $A_{0}=5 \mathrm{~cm}$ was used, and three vibrating frequencies $f_{0}=0.3,0.96$ and $1.5 \mathrm{~Hz}$ (case A, case B and case C) were used in the experiments. Herein $A_{0}$ and $f_{0}$ are the amplitude and frequency of the vibration rod.

\subsection{Vibrating characteristics of a circular cylinder with different frequency}

The motions of the cylinder displacement were recorded by using the high speed camera and PIV system with a sampling rate of $100 \mathrm{~Hz}$. Figure 3 displays the time history records of the vertical displacement of the cylinder, $\eta$ with different $f_{0}$ in still water far away from the sand bed (no plane boundary condition). The results show that the behaviors of the cylinder vibration are very distinct with different frequencies. The response amplitude $A$ $=3.05,4.53$, and $0.08 \mathrm{~cm}$ with $f_{0}=0.3,0.96$ and 1.5 $\mathrm{Hz}$, respectively. Herein the response amplitude $A$ of the vibrating cylinder is obtained by taking the average of the $10 \%$ of the highest peaks recorded for each case. If the forced vibrating frequency is close to the natural frequency of the system, $A$ would become bigger.

However, when the cylinder is close to the sand bed, the behavior of the vibrating cylinder is different from the "no plane boundary" condition. Figure 4 shows the time history records with $G_{0} / D=1.0$ when the scour hole has reached its asymptotic state. The response amplitude with $f_{0}=0.3 \mathrm{~Hz}$ reaches $5.52 \mathrm{~cm}, 81 \%$ largerthan the response amplitude of the "no plane boundary" case with the same frequency. The result reveals the important inter-dependent relationship between the bottom boundary, vibrating cylinder and scour hole.

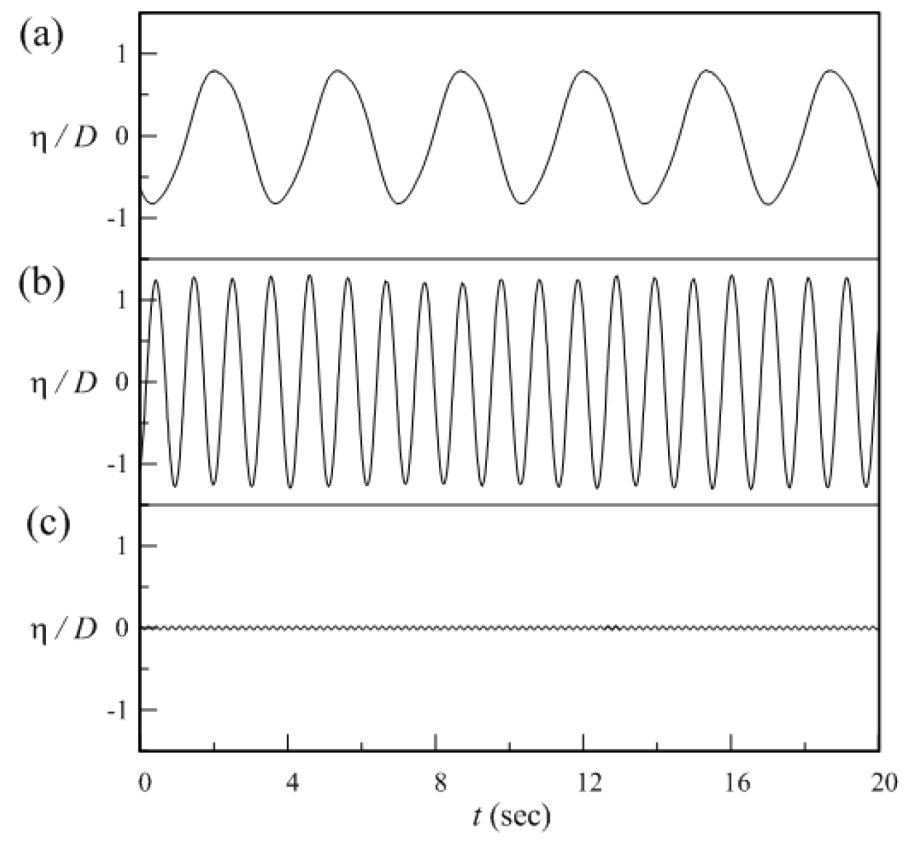

Figure 3. Time history records of the vertical displacement with different $f_{0}$ in still water (no plane boundary condition). (a) $f_{0}=0.3 \mathrm{~Hz}$; (b) $f_{0}=0.96 \mathrm{~Hz}$; (c) $f_{0}=1.5 \mathrm{~Hz}$.

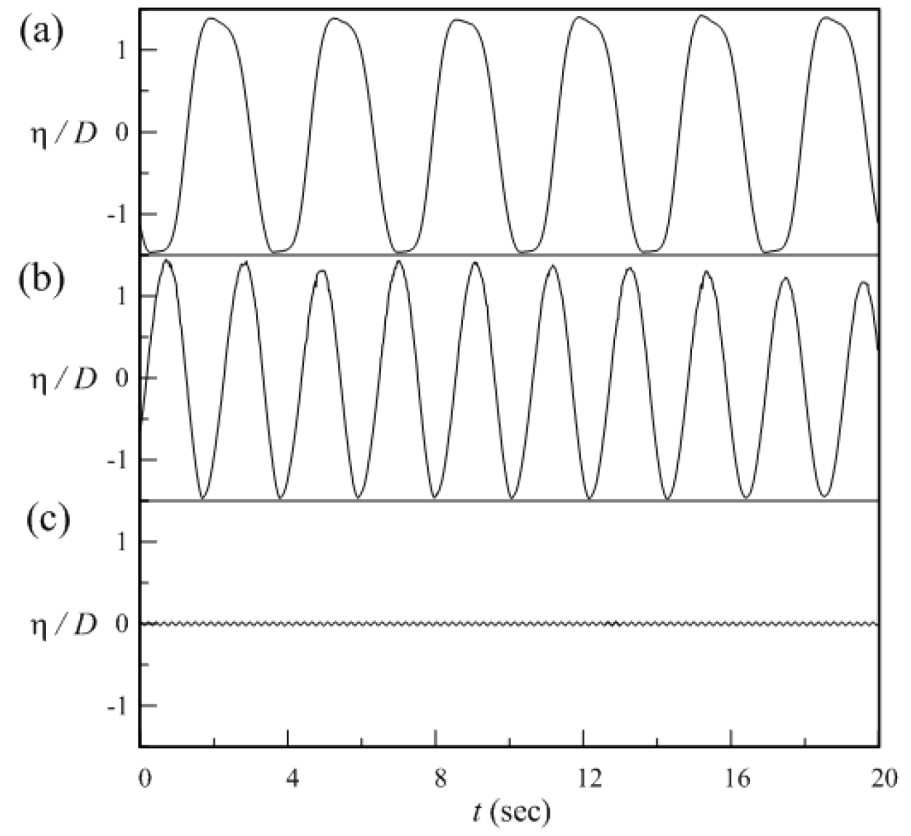

Figure 4. Time history records of the vertical displacement with different $f_{0}$ with $G_{0} / D=1.0$. (a) $f_{0}=0.3 \mathrm{~Hz}$; (b) $f_{0}=0.96$ $\mathrm{Hz}$; (c) $f_{0}=1.5 \mathrm{~Hz}$. 


\subsection{Comparison the scour hole with different vibrating frequency}

The data also show distinctly different scour hole profiles when different vibrating frequencies are imposed. Figures 5 and 6 show variations of the scour hole profiles when $f_{0}=0.3$ and $0.96 \mathrm{~Hz}$, respectively. It must be noted that no scour occurs when $f_{0}=1.5$ $\mathrm{Hz}$ because the resulting response amplitude is too small to induce any vortex formation.

When $f_{0}=0.3 \mathrm{~Hz}$, the asymptotic scour hole profile resembles a symmetrical inverted triangular cylinder, as shown in Figure 5. Figure 6 shows how the asymptotic scour profile with $f_{0}=0.96 \mathrm{~Hz}$ is markedly different from the $f_{0}=0.3 \mathrm{~Hz}$ counterpart, with the latter having a flat-bottom scour hole, somewhat resembling a trapezoidal cylinder shape, and is significantly wider. The bottom width of the scour hole is approximately 2 times the cylinder diameter. However, the depth of the scour hole $(=1.1 \mathrm{~cm})$ is smaller than the case of $f_{0}=0.3 \mathrm{~Hz}(=1.6 \mathrm{~cm})$.

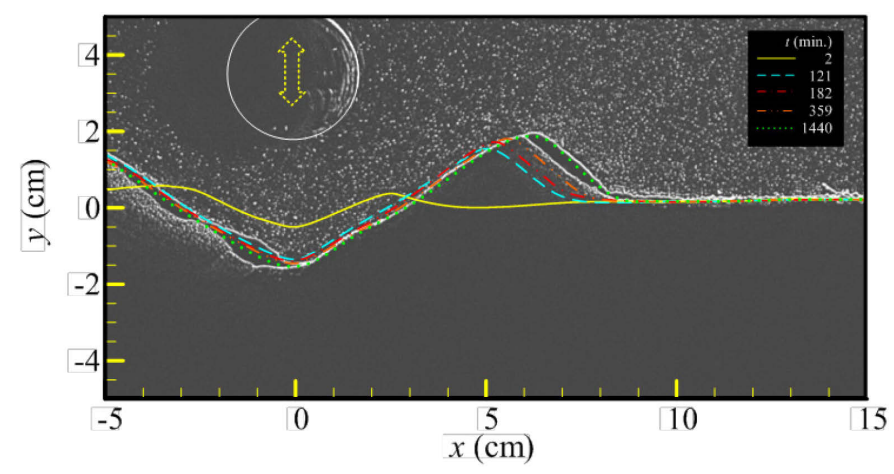

Figure 5. The profile variations of the scour hole with $f_{0}=$ $0.3 \mathrm{~Hz}, G_{0} / D=1.0$.

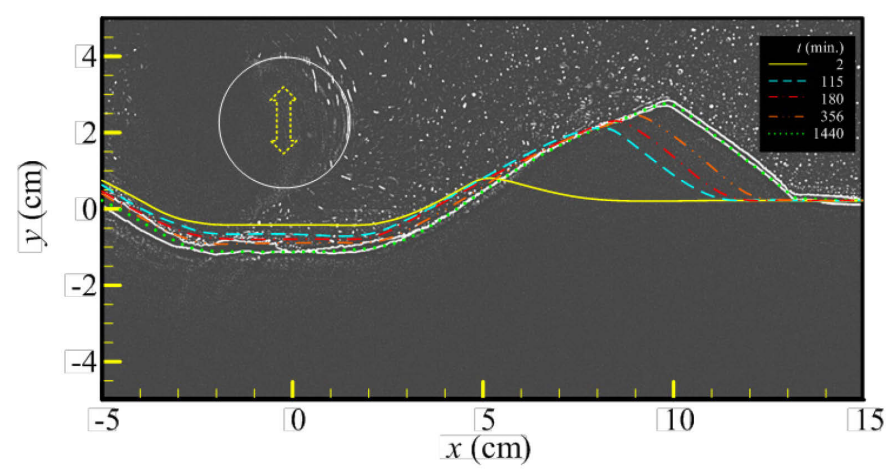

Figure 6 . The profile variations of the scour hole with $f_{0}=0.96$ $\mathrm{Hz}, G_{0} / D=1.0$.

The experimental data show that the scour hole expectedly develops quickly at their early stage of development $(t<180 \mathrm{~min}$.). This is because the vibrating cylinder bounces on the sand bed, forcing both water and sand beneath the cylinder to move toward the left and right of the cylinder. The result is the formation of a symmetrical scour hole with the ensuing sediment mounds or dunes on either sides of the cylinder. When the depth of scour hole and height of the dunes increase, it becomes more diffi- cult to "force" the bed sediment out of the hole. However, it must be stated that even when the scour hole has approached its asymptotic stage, one can still observe the bed sediment particles being "picked-up" by the movement of the cylinder, but they cannot be entrained out of the scour hole. The sediment particles merely move up and down in the scour hole.

\subsection{Variations of the velocity field in scouring process}

Figures 7 and 8 show the results of PIV measurement 1440 minutes after the commencement of the test. At this juncture, the scour hole has entered the asymptotic stage in that scouring has slowed down significantly. The phase-averaged analysis was conducted to evaluate the velocity vectors; the vorticity counters also are superimposed in the figures. Herein, the vorticity $\omega_{z}(1 / \mathrm{s})=(\Delta v / \Delta x)-(\Delta u / \Delta y)$ is the spanwise vorticity of the flow field. The red vorticity contours denote positive vorticity in the counterclockwise direction and the blue counterpart represents the negative vorticity in the clockwise direction. Moreover, it is assumed that $f_{p}$ is the primary component of the cylinder vibration, and can be represented as $\eta_{p}(t)=A_{p} \cdot \cos (t / T)$, where $A_{p}$ is the primary component amplitude; $T$ is the primary component vibrating period of the cylinder; and the time $t$ varies from 0 to $T$. In these figures, the region above the cylinder has no data because of the shadow of the cylinder. Because the flow field around the cylinder is symmetrical, the discussion below is confined to the right side $(x>0.0)$ of the cylinder.

Since the vibration speed of the cylinder is significantly different between these two cases $\left(f_{0}=0.3\right.$ and $0.96 \mathrm{~Hz}$ ), the formation and convection of vortices around the cylinder are correspondingly different. Fig. 7 shows the lower vibrating case $\left(f_{0}=0.3\right.$ $\mathrm{Hz}$ ), revealing a lower flow velocity induced by the cylinder when compared with the fast vibrating case in Fig. $8\left(f_{0}=0.96 \mathrm{~Hz}\right)$. When the cylinder is in the falling phase $(0.0<t / T<0.5)$, a pair of vortices with a similar strength but opposite in direction, are generated from the shear layers at the right and left sides of the cylinder. Figs. 7(a-c) and 8(a-c) show how the red, counter-clockwise vortex follows the cylinder and progressively increases in strength as the latter moves down to the bed. When $t / T=0.5$, the cylinder hits the sand bed and forces the sand to be suspended. In the meanwhile, the counter-clockwise vortex stays between the cylinder and the slopes of the scour hole, spewing the suspended sediment particles from the hole (see Figures 7d-e). However, in the second case (fast vibrating, $f_{0}=0.96 \mathrm{~Hz}$ ), the counter-clockwise vortex moves into the scour hole as the cylinder begins to move up (see in figure 8df). This phenomenon is probably because the counter-clockwise vortex has a stronger momentum. 
(a)

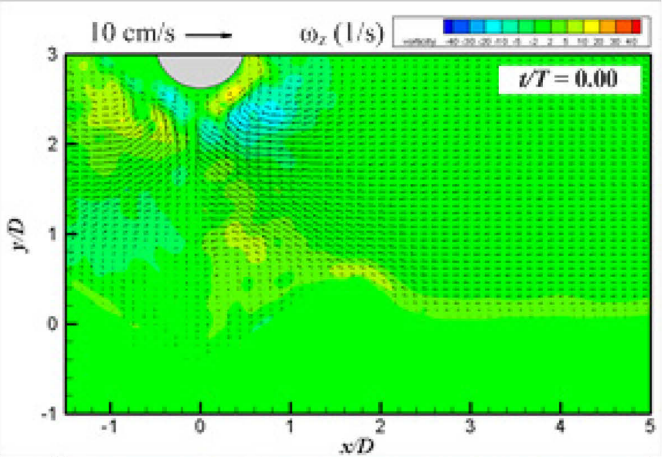

(b)

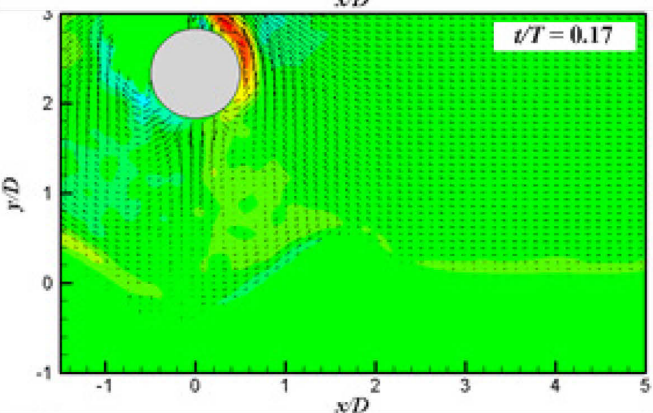

(c)

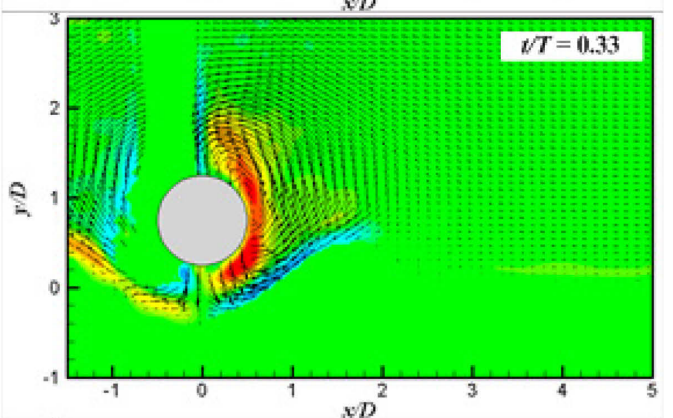

(d)

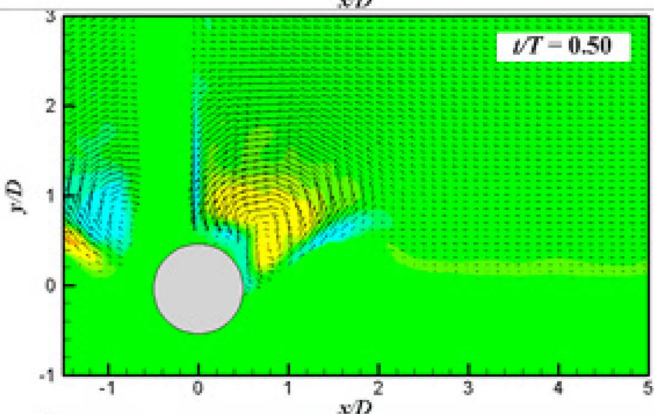

(e)

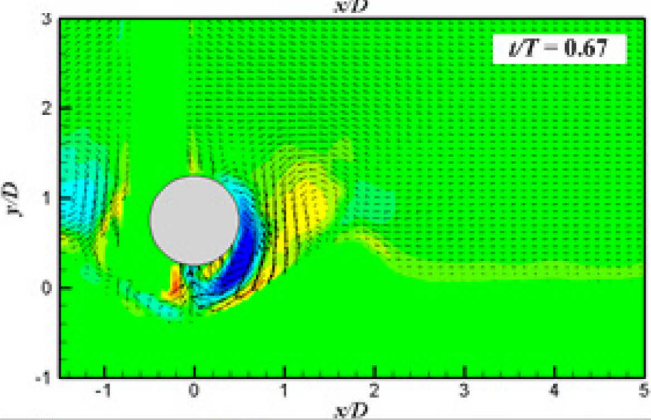

(f)

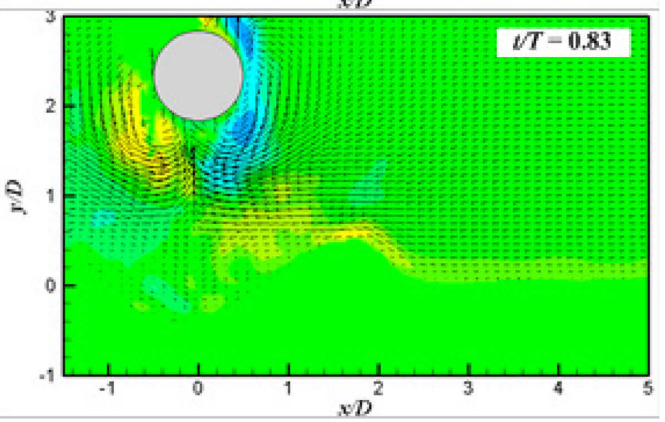

Figure 7. PIV measurement and phase average velocity vector of a forced vibrating circular cylinder with $f_{0}=0.3 \mathrm{~Hz}$ at 1440 minutes after the commencement of the test. (a)

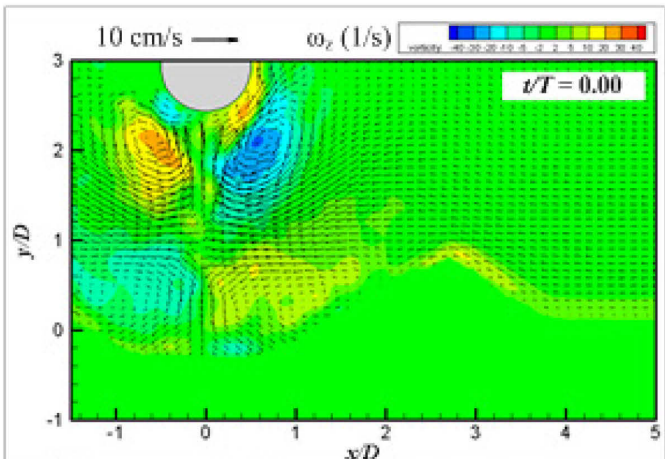

(b)

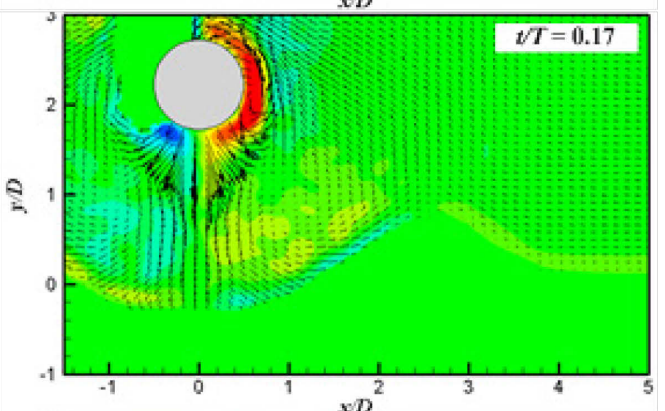

(c)

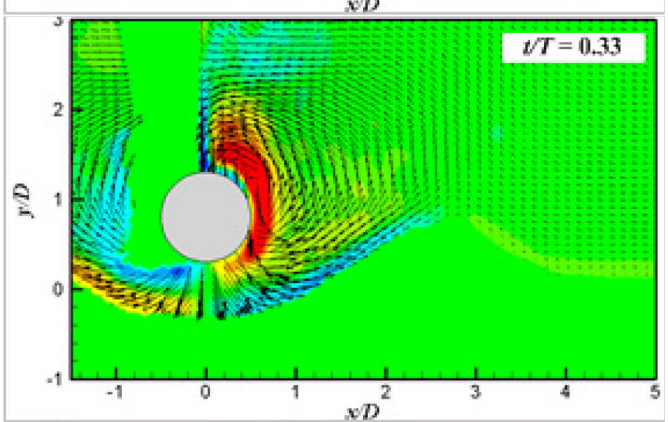

(d)

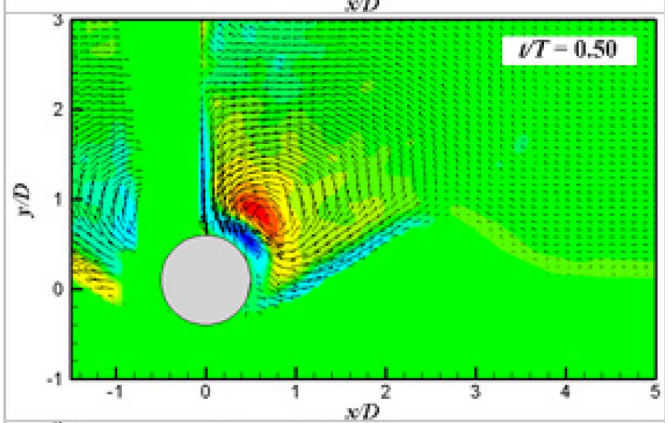

(e)

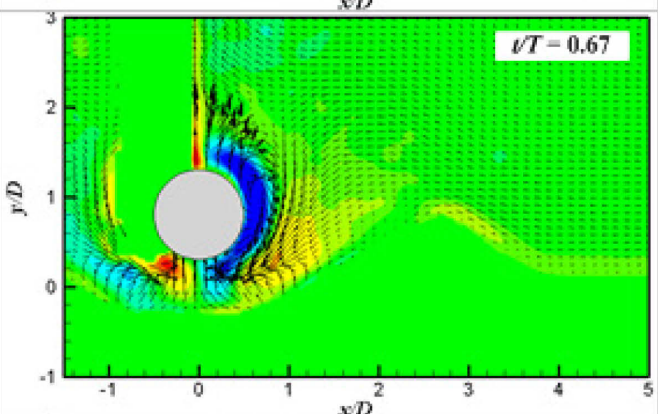

(f)

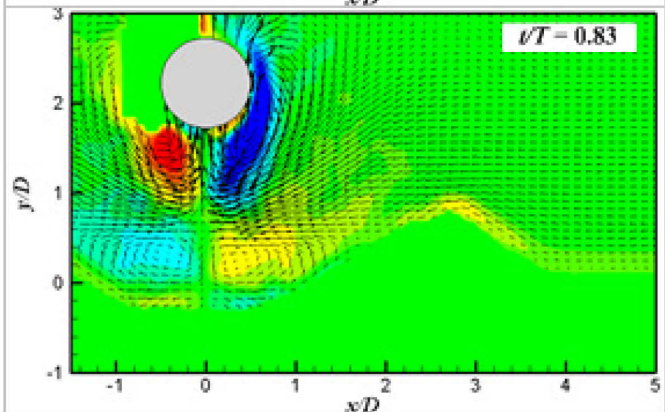

Figure 8. PIV measurement and phase average velocity vector of a forced vibrating circular cylinder with $f_{0}=0.96 \mathrm{~Hz}$ at 1440 minutes after the commencement of the test. 
Hence, the pair of vortices remains in the scour hole and is able to induce a higher bedload transport and thereby a deeper scour hole when compared with the former case. The phenomenon is likely a reason for the flat bottom of the scour hole (see Figure 6).

According to the results of the flow field and visualization, two distinctive scour holes profiles, each dependent on the vibration frequency even though their amplitude is the same, can be identified. In summary, a full understanding on the interrelationship of all the variables involved in inducing the type of scour hole still remains elusive presently; more data are still needed before one can arrive at that position.

\subsection{Mean velocity and turbulence characteristics with different vibrating frequency}

The purpose of this section is to compare the characteristics of the mean velocity field and turbulence properties around two forced vibrating cylinders with $f_{0}=0.3$ and $0.96 \mathrm{~Hz}$. Figures 9 and 10 show the results of the ensemble mean velocity fields corresponding to the flow fields of Figures 7 and 8, respectively. The turbulence properties, which include detailed distributions of the velocity fluctuations in the horizontal and vertical directions $\left(\sqrt{u^{\prime 2}}\right.$ and $\sqrt{v^{\prime 2}}$, where $u^{\prime}$ and $v^{\prime}$ are the fluctuations of $u$ and $v$ ), are also superimposed in the figures.
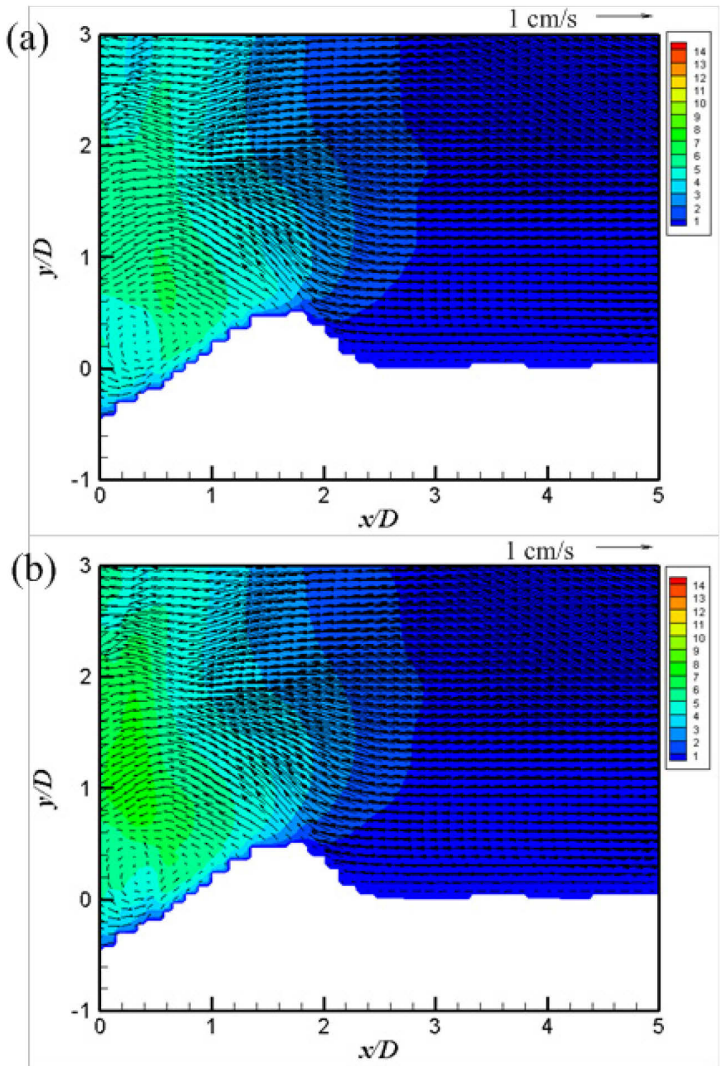

Figure 9. Ensemble mean velocity field and turbulent properties with $f_{0}=0.3 \mathrm{~Hz}$. (a) horizontal direction; (b) vertical direction (The unit for turbulence intensities is $\mathrm{cm} / \mathrm{s}$ ).
In the figures, both the mean velocity fields clearly reveal the presence of a counter-clockwise flow in the scour holes. It may be surmised that erosion in the scour hole is principally affected by the counterclockwise vortex that accompanies the descending phase of the cylinder when it moves towards the bed.

A comparison of the turbulence properties in these two cases shows that the turbulence intensity associated with the higher $f_{0}$ expectedly is stronger than its lower counterpart. Because of this stronger counter-clockwise vortex (in case B), it persists to impinge onto the scour hole even during the ascending phase of the pipeline. This probably is the reason why this part of the scour hole is plane.
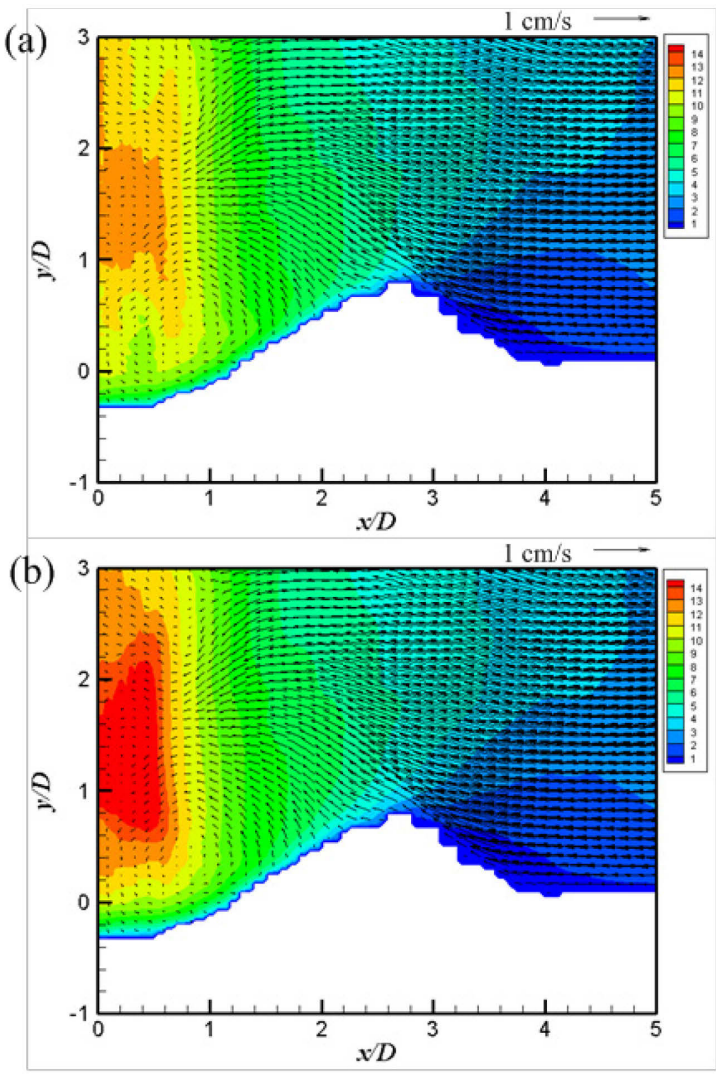

Figure 10. Ensemble mean velocity field and turbulent properties with $f_{0}=0.96 \mathrm{~Hz}$. (a) horizontal direction; (b) vertical direction (The unit for turbulence intensities is $\mathrm{cm} / \mathrm{s}$ ).

\section{CONCLUSIONS}

This study is an attempt to investigate the effect of the forced vibration induced by an elastically mounted circular cylinder on scour in still water. Only one gap ratio $G_{0} / D=1.0$, with forced vibration amplitude $A_{0} / D=1.43$, and three forced vibration frequency $f_{0}=0.3,0.96$ and $1.5 \mathrm{~Hz}$ were tested. The flow field and turbulence properties around the vibrating cylinder with different $f_{0}$ measured using flow visualization and PIV also were conducted to help explain the different types of scouring process. 
The main findings are summarized as follows:

1. The study shows that the characteristics of vibration of the cylinder elastically mounted on a forced vibrating rod will become different when the cylinder is close to the sand bed $\left(G_{0} / D=1.0\right)$. This behavior reveals the important effect of the proximity from the bottom boundary on the interaction between the vibrating cylinder and scour hole.

2. The speed of vibration or vibration frequency plays an important role in the formation of the scour hole. A scour hole with the profile of an inverted triangle is formed when the frequency is small. On the other hand, the scour hole has the profile of a trapezoidal cylinder when the frequency increases. The reason for the difference is probably due to the stronger vortex and turbulence intensities associated with higher frequency.

3. Two distinct types of scour process, which dictates whether the vortices impinge on the scour hole or not, can be observed with different vibration frequencies although a precise understanding of this process remains uncertain presently. Additional data are needed to better understand this phenomenon.

\section{ACKNOWLEDGMENTS}

The authors gratefully acknowledge the financial support provided by Singapore Maritime Institute (SMI) and EMAS AMC, under the SMI Deepwater Technology R\&D Programme.

\section{REFERENCES}

Aubeny, C.P., Gaudin, C. \& Randolph, M.F. 2008. Cyclic tests of model pipe in kaolin. Proceeding of offshore technology conference, Paper OTC 19494.

Clukey, E.C., Young, A.G., Dobias, J.R. \& Garmon G.R. 2008. Soil response and stiffness laboratory measurements of SCR pipe/soil interaction. Proceeding of offshore technology conference, Paper OTC 19303.

Cowen, E.A. \& Monismith, S.G. 1997. A hybrid digital particle tracking velocimetry technique. Experiments in Fluids 22: 199-211.

Hodder, M.S., White, D.J. \& Cassidy, M.J. 2009. Effect of remolding and reconsolidation on the touchdown stiffness of a steel catenary riser: Observations from centrifuge modeling. Proceeding of offshore technology conference, Paper OTC 19871

Hodder, M.S. \& Byrne, B.W. 2010. 3D experiments investigating the interaction of a model SCR with the seabed. Applied Ocean Research 32: 146-157.

Hsieh, S.C. 2008. Establishment of high time-resolved PIV system with application to the characteristics of a near wake flow behind a circular cylinder. Ph.D. thesis, National Chung Hsing Univ., Taiwan.

Hu, H.J.E., Leung, C.F., Chow, Y.K. \& Palmer, A.C. 2009. Centrifuge modelling of SCR vertical motion at touchdown zone. Ocean Engineering 38: 888-899.
Langford, T. \& Aubeny, C.P. 2008. Model tests for steel catenary riser in marine clay. Proceeding of offshore technology conference, Paper OTC 19495.

Li, F.Z., Dwivedi, A., Low, Y.M., Hong, J.H. \& Chiew, Y.M., 2013. Experimental investigation on scour under a vibrating catenary pipe. Journal of Engineering Mechanics, ASCE 139(7): 868-878.

Sumer, B.M. \& Fredsøe, J. 1997. Hydrodynamics around Cylindrical Structures. World Scientific.

Westgate, Z.J., White, D.J. \& Randolph, M.F. 2013. Modelling the embedment process during offshore pipe-laying on finegrained soils. Can. Geotech. J. 50: 15-27. 